\title{
Strategies for mitigating an influenza pandemic
}

\author{
Neil M. Ferguson ${ }^{1}$, Derek A. T. Cummings ${ }^{2}$, Christophe Fraser ${ }^{1}$, James C. Cajka ${ }^{3}$, Philip C. Cooley ${ }^{3}$ \\ \& Donald S. Burke ${ }^{2}$
}

Development of strategies for mitigating the severity of a new influenza pandemic is now a top global public health priority. Influenza prevention and containment strategies can be considered under the broad categories of antiviral, vaccine and nonpharmaceutical (case isolation, household quarantine, school or workplace closure, restrictions on travel) measures ${ }^{1}$. Mathematical models are powerful tools for exploring this complex landscape of intervention strategies and quantifying the potential costs and benefits of different options ${ }^{2-5}$. Here we use a large-scale epidemic simulation $^{6}$ to examine intervention options should initial containment ${ }^{6,7}$ of a novel influenza outbreak fail, using Great Britain and the United States as examples. We find that border restrictions and/or internal travel restrictions are unlikely to delay spread by more than 2-3 weeks unless more than $99 \%$ effective. School closure during the peak of a pandemic can reduce peak attack rates by up to $40 \%$, but has little impact on overall attack rates, whereas case isolation or household quarantine could have a significant impact, if feasible. Treatment of clinical cases can reduce transmission, but only if antivirals are given within a day of symptoms starting. Given enough drugs for $50 \%$ of the population, household-based prophylaxis coupled with reactive school closure could reduce clinical attack rates by $40-50 \%$. More widespread prophylaxis would be even more logistically challenging but might reduce attack rates by over $75 \%$. Vaccine stockpiled in advance of a pandemic could significantly reduce attack rates even if of low efficacy. Estimates of policy effectiveness will change if the characteristics of a future pandemic strain differ substantially from those seen in past pandemics.

We parameterize an individual-based simulation model of pandemic influenza transmission ${ }^{6}$ for Great Britain and the United States using high-resolution population density data $^{8}$ and data on travel patterns (see Supplementary Information). We extend the model by incorporating realistic seeding of infection (via international travel) in the modelled countries, and by explicitly modelling air travel within the United States (air travel being relatively insignificant in Great Britain due to its much smaller size).

The model represents transmission in households, schools and workplaces, and the wider community. For the United States, best estimates are that $30 \%$ of transmission occurs within the household, and $70 \%$ outside the household (see Supplementary Information). Of the latter $70 \%$, we assume that $33 \%$ occurs in the general community and $37 \%$ in schools and workplaces. To reproduce the higher attack rates seen in children in past pandemics, per-capita contact rates in schools were assumed to be double that in workplaces $^{6}$. These assumptions affect estimates of the impacts of certain targeted control policies; for example, if school/workplace transmission were assumed to account for $50-60 \%$ of transmission, then policies such as school closure and socially targeted prophylaxis would be more effective (see Supplementary Information).
Acquiring more quantitative data on transmission in different social contexts should therefore be a priority.

We estimated the reproduction number ${ }^{9}$ for pandemic influenza, $R_{0}$, to have a value of $1.7-2.0$ for the first wave of the 1918 pandemic, as determined from city-level mortality data (see Supplementary Information). In 1957, epidemic growth rates were less, with UK national data giving $R_{0}$ values of 1.5-1.7 (see Supplementary Information). Inter-pandemic data give a value of $R_{0} \approx 1.7$ (see Supplementary Information). We therefore examine values of $R_{0}$ in the range 1.4 to 2 , particularly focusing on how conclusions differ for 'moderate' $\left(R_{0}=1.7\right)$ and 'high' $\left(R_{0}=2.0\right)$ transmission scenarios. Because the natural history of infection for human cases of avian $\mathrm{H} 5 \mathrm{~N} 1$ infection have to date been much more extended (and severe) than normal human influenza ${ }^{10,11}$, we also examine sensitivity to assumptions about the duration of infectiousness. We do not assume any spontaneous change in the behaviour of uninfected individuals as the pandemic progresses, but note that behavioural changes that increased social distance together with some school and workplace closure occurred in past pandemics ${ }^{12}$ (see Supplementary Information) and might be likely to occur in a future pandemic even if not part of official policy. Data on respiratory infection incidence in Hong Kong during the severe acute respiratory syndrome (SARS) epidemic supports this view ${ }^{13}$. Such spontaneous changes in population behaviour might more easily reduce peak daily case incidence than overall cumulative attack rates (see Supplementary Information). The peak incidence of the high transmissibility scenario examined here is therefore probably a worst case.

We assume that 50\% (see Supplementary Information for sensitivity analysis) of those infected are ill enough to be classified as clinical cases (that is, those requiring and seeking medical care), consistent with known patterns for seasonal influenza. In reality, a spectrum of disease severity will be seen in a pandemic. Given the lack of data on the virulence of the next pandemic strain, the impact of antivirals on mortality, and the effects of improvements in general medical care, we examine the impact of interventions on clinical attack rates rather than mortality.

Figure 1 shows the expected pattern of spread of an influenza pandemic in Great Britain and the United States, for moderate and high transmissibility scenarios (Fig. 1a, b). The epidemic peaks some 50-65 days after the first case in the country for Great Britain, and some 60-80 days after the first case for the United States. Peak daily case incidence is slightly higher in Great Britain than the United States due to the smaller spatial scale of Great Britain and hence the more synchronized regional outbreaks. But peak local incidence in the United States is comparable with Great Britain, and cumulative incidence over the epidemic is identical for both countries. For the moderate and high transmissibility scenarios, $55 \%$ and $68 \%$ of the population are infected, respectively, giving cumulative clinical attack rates of $28 \%$ and $34 \%$ (namely, 84 million or 100 million

${ }^{1}$ Department of Infectious Disease Epidemiology, Faculty of Medicine, Imperial College London, St Mary's Campus, Norfolk Place, London W2 1PG, UK. ${ }^{2}$ Department of International Health, Johns Hopkins Bloomberg School of Public Health, 615 North Wolfe Street, Baltimore, Maryland 21205, USA. ${ }^{3}$ RTI International Inc., P.O. 12194, 3040 Cornwallis Rd, Research Triangle Park, North Carolina 27709, USA. 
sick individuals in the United States). Absenteeism from work is, in reality, a complex function of case incidence, but can be approximated by case incidence multiplied by total numbers of work days lost per case. Assuming 7 work days lost per clinically ill case, nationwide absenteeism would peak at $10 \%$ in Great Britain and at $9 \%$ in the United States for the moderate transmissibility scenario. Local absenteeism can be substantially higher (each national epidemic is composed of slightly desynchronized local epidemics).

The dependence of the timing of the epidemic peak and the clinical attack rate on $R_{0}$ is shown in Fig. 1c. There is considerable stochastic variation in the timing of the first few cases, associated with the randomness of the disease importation process (Fig. 1d). But peak timing is much less variable. The rate at which infected persons enter a country from overseas will affect the likely rate of spread within a country. In the absence of a global simulation of pandemic spread, we modelled the global epidemic with a simple compartmental transmission model, and used international travel data to estimate the numbers of infected persons expected to arrive in either the United States or Great Britain per day (see Supplementary Information). The global model used was non-spatial, so it was not possible to examine how the country of origin of a pandemic would affect how quickly and in which order the United States and Great Britain would be infected. But use of even a non-spatial transmission model is preferable to simply assuming a fixed starting number of infected persons or a constant importation rate through time, as the expected exponential increase in imported infections over time significantly speeds spatial spread within the United States and Great Britain compared with assuming a single point seeding event.

Little spatial structure is seen in the pattern of pandemic establishment and spread in Great Britain (see Supplementary Videos) due to its relatively small size and frequent long-range travel. In the United States, more structure is apparent: early spread is focal around seed infections (typically in urban centres) imported from overseas, but rapidly becomes almost homogenously distributed across the whole population (Fig. 1e and Supplementary Videos).

After the identification of a new human-to-human transmissible influenza A virus strain anywhere in the world, attempts to prevent spread to unaffected countries are likely, and international travel may be restricted ${ }^{14-16}$. Here we examine the impact of the border controls imposed by the United States or Great Britain to reduce numbers of inbound travellers (the results are very similar for both countries). Figure 2 a shows how a $90 \%, 99 \%$ or $99.9 \%$ reduction in imported infections might delay the peak of the US pandemic by $1.5,3$, or 6 weeks, respectively (comparable delays would be expected for other Western countries given the similar mobility of their populations). To put these reductions into context, the 2003 SARS crisis resulted in an $80 \%$ reduction in travel to and from Hong Kong ${ }^{17}$. The magnitude of the impact of border controls is governed by the rate at which global infection prevalence increases. A tenfold reduction in numbers of visitors delays arrival of infection for approximately as long as it takes global prevalence to increase tenfold to compensate- 12.5 days using the global model assumed here.

We next examined the effectiveness of travel restrictions within the United States at slowing national spread. If border controls are not in place, then external seeding from infected international travellers overwhelms the effect of within-country restrictions, only delaying the peak of the epidemic by less than 1 week. If combined with $99.9 \%$ effective border controls, blanket reductions in non-local travel achieve little in delaying the peak of the epidemic (Fig. 2b), but do reduce the peak attack rate substantially and spread the epidemic over a much longer time period (see Supplementary Information). Eliminating travel in and out of affected regions can delay spread by up to 2 weeks beyond the 6 weeks achieved by border restrictions alone. The delay achievable in Great Britain is half this. In the United States, closing airports for domestic travel also has little impact, due to the substantial volume of long distance travel by road. Internal restrictions must be highly effective to have much impact-although $90 \%$ effective internal restrictions can still have some effect, $75 \%$ effectiveness has almost none.

Once a new pandemic virus starts to be transmitted in a country, interventions must be targeted for maximum impact. Applying the type of intensive control strategies envisaged for containing a pandemic at source ${ }^{6}$ is impractical as infection will constantly be reseeded in a country by visitors (see Supplementary Information). Clinical cases are clearly then the first priority for any more-targeted approach, as prompt treatment with antivirals reduces clinical severity and infectiousness ${ }^{18}$ (see Supplementary Information). Our results indicate that only very rapid treatment can significantly

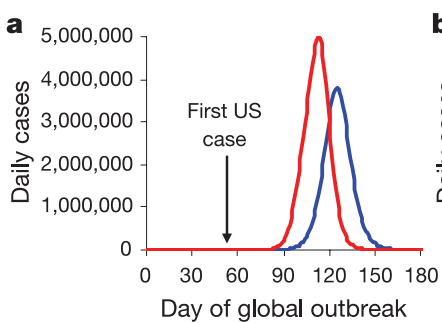

e
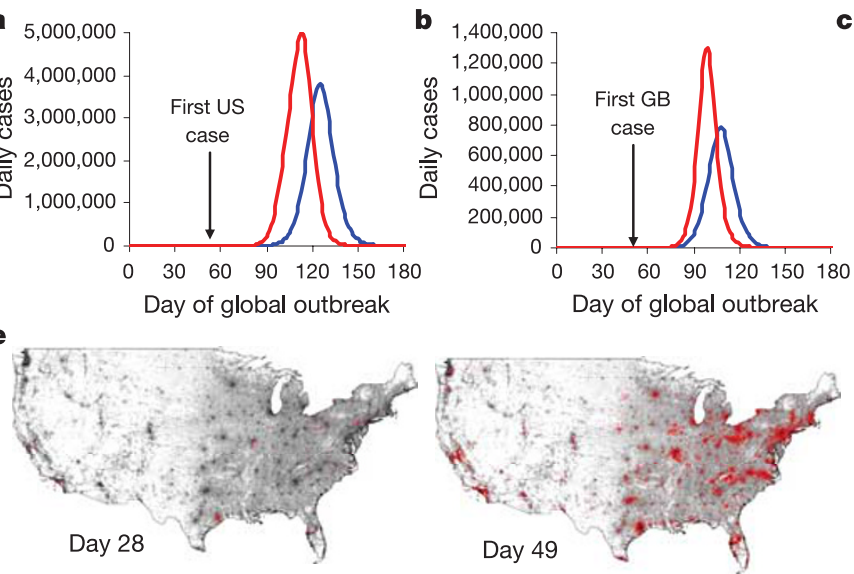

Figure 1 | Baseline pandemic dynamics. a, Clinical case incidence per day for the US pandemic (single realization shown) for high (red) and moderate (blue) transmissibility scenarios, assuming a generation time of 2.6 days, and that $50 \%$ of infected people are ill enough to be classified as clinical cases. Infection is seeded in the country as a function of the expected importation of infection from overseas derived from a simple global model of pandemic spread and available travel data (see Supplementary Information). Assumed population size of the United States was 300 million. Timing is shown both as days from the first case globally, and as days from the first case in the country. b, As a, but for Great Britain (modelled
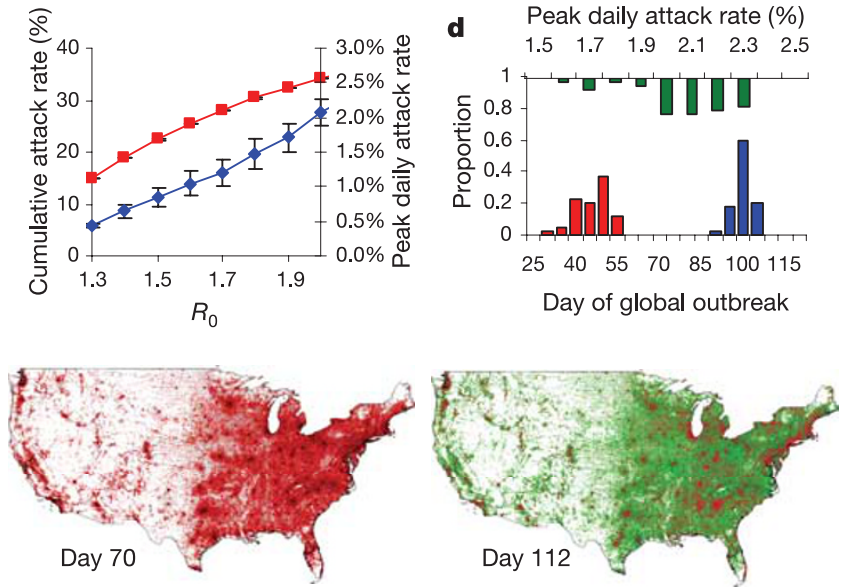

population size 58.1 million). c, Cumulative (red) and peak daily (blue) clinical attack rates as a function of $R_{0}$ for Great Britain, averaged over 40 model realizations. Error bars show standard deviations. d, Histogram showing stochastic variability (across 40 model realizations) in timing of initial case (red), peak of epidemic (blue) and peak attack rate (green) for Great Britain $\left(R_{0}=2.0\right)$. e, Snapshots of the extent of spread of the US pandemic (moderate transmissibility) at four time points. Greyscale indicates population density; red indicates areas with infective cases; and green indicates areas where the pandemic is over. See Supplementary Information for full parameter and model details. 
reduce transmission (Fig. 2c, d), because cases are at their most infectious soon after symptoms develop (see Supplementary Information). For the high transmissibility scenario, same-day treatment of $90 \%$ of cases reduces cumulative attack rates from $34 \%$ to $29 \%$ and peak daily attack rates from $1.9 \%$ to $1.6 \%$, with an antiviral stockpile sufficient to treat $25 \%$ of the population (the size many countries have ordered ${ }^{19}$ ) being adequate to implement the policy. If treatment is delayed by 1 day, the cumulative attack rate for the high transmissibility scenario increases to $32 \%$ (meaning that a $29 \%$ stockpile is needed), and the peak daily attack rate to $1.9 \%$. The impact of treatment on the peak daily attack rate at the height of the epidemic is always greater than that on overall attack rates. Assuming that more than $50 \%$ of infections result in clinical illness requiring treatment would increase the required stockpile (see Supplementary Information). A real threat to the effectiveness of antiviral-based mitigation policies would be if resistant strains arose with transmissibility close to the wild-type level ${ }^{20}$. Such strains have not yet been detected, but resistance monitoring during a pandemic will be essential.

Like treatment, rapid case isolation reduces the infectiousness of the targeted individual and can have a similar or greater impact (Fig. 2e) - reducing cumulative attack rates from $34 \%$ to $27 \%$ for $R_{0}=2.0$ if $90 \%$ of cases are isolated. Conservatively, we assume that clinical cases have a reduced contact rate in their school or workplace and with the wider community (see Supplementary Information) even in the absence of a policy of case isolation. Case isolation here is assumed to reduce all contact rates by a further uniform fraction,
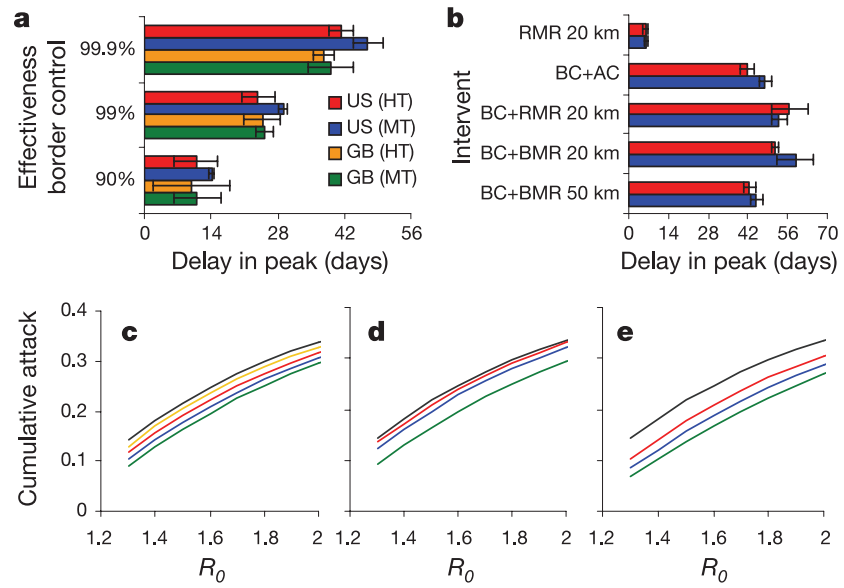

Figure 2 Impact of travel restrictions and case-targeted policies. a, Delay caused by $90 \%, 99 \%$ and $99.9 \%$ reduction of imports of infection from day 30 of the global pandemic onwards on peak timing for GB and US pandemics for high (HT) and moderate (MT) transmissibility scenarios. Mean \pm s.d. of 5-20 realizations is shown in both $\mathbf{a}$ and $\mathbf{b}$. $\mathbf{b}$, Delay in the peak of the US pandemic caused by internal travel restrictions for high (red) and moderate (blue) transmissibility. All policies are assumed to start after $\mathbf{5 0}$ cases have been diagnosed in the country. AC, all airports in the United States are closed to internal traffic; $\mathrm{BC}$, border controls (external imports are reduced by $99.9 \%$ ); BMR, blanket movement restrictions (journeys over 20 or $50 \mathrm{~km}$ from the home are eliminated); RMR, reactive movement restrictions (a 20-km exclusion zone is established around every diagnosed case, with overlapping zones being merged, and movement in and out of the exclusion zone is eliminated). c, Cumulative clinical attack rates for same day antiviral treatment policy, shown as a function of $R_{0}$. From highest to lowest, the different curves represent $0 \%, 30 \%, 50 \%, 70 \%$ and $90 \%$ of cases treated. Results for Great Britain are shown (US results are identical). d, As c, but showing dependence on delay (in days) between symptom onset and treatment when $90 \%$ of cases are treated (curves, from highest to lowest, are for no treatment, 2 day, 1 day and 0 day delay). e, As c, but showing effect of same day case isolation causing a $90 \%$ reduction in contacts (from highest to lowest, curves are for $0 \%, 50 \%, 70 \%$ and $90 \%$ of cases isolated). including in the household-the policy is less effective if household contacts are not reduced.

Being a member of a household containing an influenza case is in fact the largest single risk factor for being infected oneself ${ }^{2,22}$. Two policies targeting households are available (Fig. $3 \mathrm{a}-\mathrm{c}$ ): prophylaxis using antiviral drugs, and quarantine (requesting persons in households with infected cases to remain at home). Antiviral prophylaxis of household members is effective in reducing cumulative attack rates by at least onethird and peak attack rates by a half (Fig. 3a, b), but requires an antiviral stockpile large enough to treat $46 \%$ or $57 \%$ of the population for the moderate and high transmissibility scenarios, respectively. Household quarantine is also effective at reducing attack rates in the community (indeed, for low $R_{0}$ values, pandemic spread can be dramatically slowed), but only if compliance is high. However, given the expected increases in contact rates within the household which would result, a household quarantine policy might pose ethical dilemmas unless excellent infection control was implemented. A combined policy of household quarantine and household prophylaxis is arguably more feasible and could be highly effective.

School closure (Fig. 3d-f) causes a small reduction in cumulative attack rates, but a more substantial reduction in peak attack rates (of up to $40 \%$ ). Such a reduction in peak incidence could mitigate stresses on healthcare systems and absenteeism in the critical workforce. Closure of $50 \%$ of workplaces can enhance the impact of school closure, but at higher economic cost (Fig. 3d-f). For a socially targeted prophylaxis policy, best use is made of drugs by targeting classmates or close work colleagues rather than the entire population of a school
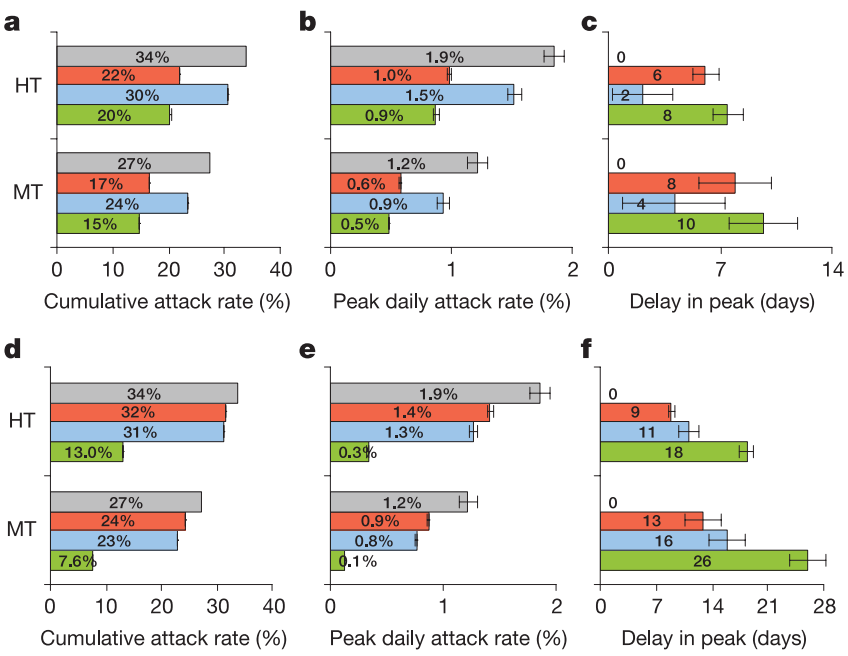

Figure 3 Impact of household/socially targeted policies. Results shown for high (HT) and moderate (MT) transmissibility scenarios in the United States. a-c, Cumulative clinical attack rate (a), peak attack rate (b) and delay in peak achieved by policy (c) (as a percentage of total population size). Values in the absence of interventions are shown in grey. Three household policies are shown: red, treating $90 \%$ of clinical cases and applying prophylaxis to their households the day after symptoms start; blue, voluntary quarantine of households identified with a clinical case in the home for 14 days- $50 \%$ of households are assumed to comply with the policy, and in these, external contact rates are reduced by $75 \%$ and withinhousehold contact rates assumed to increase by $100 \%$; green, combination of the previous two (red and blue) policies. d-f, As a-c, but for three school/ workplace-targeted policies: red, reactive school closure (that is, closing $100 \%$ of schools (and 10\% of workplaces) from the day after the first case in pupils or staff is detected until up to 3 weeks after the last case in the school) - contact rates in affected households are assumed to increase by $50 \%$ and community contact rates in absent staff/pupils by $25 \%$; blue, as above (red) but assuming $50 \%$ of workplaces close; green, as household prophylaxis policy shown in a-c but adding prophylaxis of $90 \%$ of members of the same school class or work group as treated cases. Error bars show standard deviation of between 5 and 20 realizations. 

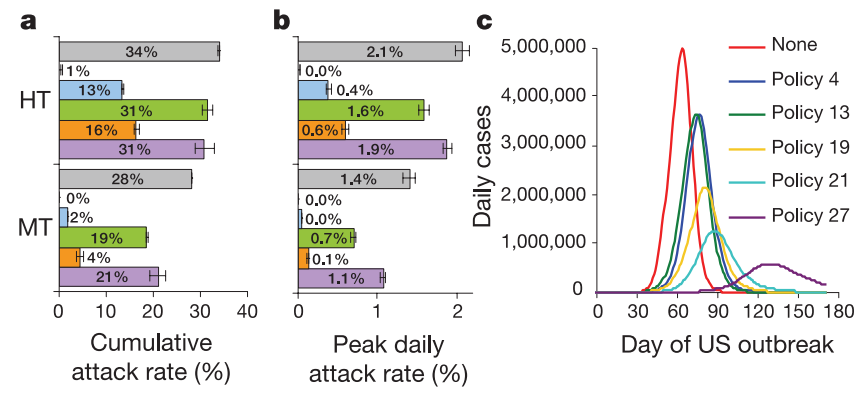

Figure 4 | Impact of vaccination and combination strategies. Results for the United States are shown. a, b, As Fig. 3a, b, but for a policy of mass vaccination assuming $1 \%$ of the population can be vaccinated per day from day 30 (red, second bar down), 60 (blue), 90 (green) after the first worldwide case, with $0-16$ yr olds vaccinated first. Results for random (non-age prioritized) vaccination are also shown (orange), and for a policy of prioritizing those over $60 \mathrm{yr}$ old (purple), both policies assuming that $1 \%$ of the population is vaccinated per day from day 60 . Values in the absence of interventions are shown in grey. There is almost no effect of vaccination if started after 120 days. For comparison, the first US case is seen on day 47, and an untreated high transmissibility US pandemic peaks on day 113, on average. c, Example epidemic curves (for US high transmissibility scenario) for a selection of combination policies from Supplementary Table SI1. Policies shown are: number 4 (50\% household quarantine plus reactive school closure); 12 (as number 4 plus $50 \%$ next-day case treatment); 13 ( $90 \%$ case treatment plus reactive school closure); 19 (as number 13 plus household prophylaxis); 21 (as number 19 plus pre-vaccination of $20 \%$ of the population, prioritizing children); and 27 (as number 19 plus prophylaxis of $80 \%$ of school classmates and close work colleagues plus $99 \%$ effective border controls).

or workplace (see Supplementary Information). School/workplace prophylaxis could have a dramatic impact on attack rates (Fig. 3d-f), but this requires antiviral stockpiles of $72 \%$ or $102 \%$ of population size for the moderate and high transmissibility scenarios, respectively.

We now explore how rapidly vaccine needs to be deployed to have a significant impact. We assume a single dose vaccine giving protection in 2 weeks that reduces susceptibility by $70 \%$, and infectiousness and probability of becoming a clinical case of those still getting infected by $30 \%$ and $50 \%$, respectively. Vaccination at the rate of $1 \%$ of the population per day would need to begin within 2 months (Fig. 4a) of the initial global outbreak (or equivalently, at approximately the same time as the first cases are seen in the United States or Great Britain)_substantially faster than is possible using current vaccine technologies. A delay of 4 months from the start of the global pandemic would mean that the initial epidemic would be largely over by the time most of the modelled populations could be vaccinated. If two doses were required 1 month apart to achieve the same level of protection, then vaccination needs to start a month earlier still for the same impact. However a $20 \%$ stockpile of pre-prepared vaccine against the source avian virus could have a substantial impact on attack rates (Fig. $4 \mathrm{c}$ and Supplementary Table SI 1), even if its efficacy was less than a vaccine matched against the pandemic strain (we assume only a $30 \%$ reduction in susceptibility conferred). A staged vaccination programme has maximum effect on reducing transmission if children are vaccinated first, because school-age children have the highest transmission rates (Fig. 4a). Vaccinating the elderly first gives the lowest impact on transmission.

In reality, interventions will be applied in combination to reduce transmission in different social compartments simultaneously. We explore a representative sample of combination policies in the Supplementary Information, and show a subset of these in Fig. 4c. In addition to the impact of pre-vaccination, a number of other conclusions stand out. Household quarantine is potentially the most effective social distance measure, but only if compliance with the policy is good. Reactive school closure has limited impact on overall attack rates, but can enhance other policies. For the high transmissibility scenario only policies using $>99 \%$ border controls can delay spread by enough to enable pandemic vaccine to reduce attack rates substantially (vaccine is assumed to be produced from month 4 of the global epidemic).

The transmissibility of a future pandemic virus is uncertain, so we explored a number of scenarios here. It is also uncertain whether the generation time of a future pandemic strain would resemble that of typical human influenza, given the greater disease severity and more protracted course seen in patients infected with the avian $\mathrm{H} 5 \mathrm{~N} 1$ virus $^{10,11}$. Paradoxically, a virus that caused more severe and extended disease might be easier to control so long as $R_{0}$ was still comparable with previous pandemic strains. A further uncertainty is the proportion of transmission occurring in schools and workplaces. If the proportion is higher than we assume, the impact of school closure and school/workplace-targeted prophylaxis would increase. The impact of case isolation and household quarantine also depends on the assumptions made about the extent to which clinical cases and other members of the household reduce their contact rates while they are ill. Finally, the proportion of infections identified as clinical cases during the pandemic (here assumed to be 50\%) can affect policy outcome significantly. See the Supplementary Information for discussion of these issues.

Lack of data prevent us from reliably modelling transmission in the important contexts of residential institutions (for example, care homes, prisons) and health care settings; detailed planning for use of antivirals, vaccines and infection control measures in such settings are needed, however. We do not present projections of the likely impact of personal protective measures (for example, face masks) on transmission, again due to a lack of data on effectiveness ${ }^{12}$.

Although more detailed model validation and parameter estimation using data from past pandemics should be a priority for future research, it will be impossible to predict the exact characteristics of any future pandemic virus. If transmissibility is found to be more similar to the levels seen in 1968 or 1957 rather than in 1918, global spread will be slower, and all the non-travel-related control policies examined here will have substantially greater impact. If the duration of disease and shedding were to be extended compared with typical human influenza ${ }^{10,11}$, then spread might be slower still, offering more potential for intervention. It will be imperative to collect the most detailed data on the clinical and epidemiological characteristics of a new virus and the impact of control measures early in the emergence of a pandemic, and to analyse those data in real time $e^{23,24}$ to allow interventions to be tuned to match the virus the world faces.

\section{Received 25 January; accepted 11 April 2006.}

Published online 26 April 2006.

1. World Health Organisation. WHO global influenza preparedness plan. 〈http:// www.who.int/csr/resources/publications/influenza/GIP_2005_5Eweb.pdf) (2005).

2. Ferguson, N. M., Donnelly, C. A. \& Anderson, R. M. Transmission intensity and impact of control policies on the foot and mouth epidemic in Great Britain. Nature 413, 542-548 (2001)

3. Keeling, M. J. et al. Dynamics of the 2001 UK foot and mouth epidemic: stochastic dispersal in a heterogeneous landscape. Science 294, 813-817 (2001).

4. Riley, S. et al. Transmission dynamics of the etiological agent of SARS in Hong Kong: Impact of public health interventions. Science 300, 1961-1966 (2003).

5. Lipsitch, M. et al. Transmission dynamics and control of severe acute respiratory syndrome. Science 300, 1966-1970 (2003)

6. Ferguson, N. M. et al. Strategies for containing an emerging influenza pandemic in Southeast Asia. Nature 437, 209-214 (2005).

7. Longini, I. M. Jr et al. Containing pandemic influenza at the source. Science 309, 1083-1087 (2005)

8. Oakridge National Laboratory. Landscan global population data. 〈http:// www.ornl.gov/sci/gist/landscan $\rangle$ (2003).

9. Anderson, R. M. \& May, R. M. Infectious Diseases of Humans; Dynamics and Control (Oxford Univ. Press, Oxford, 1991).

10. Chotpitayasunondh, T. et al. Human disease from influenza A (H5N1), Thailand, 2004. Emerg. Infect. Dis. 11, 201-209 (2005).

11. The Writing Committee of the WHO Consultation on Human Influenza A/H5N1. Current concepts-Avian influenza A (H5N1) infection in humans. N. Engl. J. Med. 353, 1374-1385 (2005). 
12. World Health Organisation Writing Group. Non-pharmaceutical interventions for pandemic influenza, national and community measures. Emerg. Infect. Dis. 12, 88-94 (2006).

13. Lo, J. Y. et al. Respiratory infections during SARS outbreak, Hong Kong, 2003. Emerg. Infect. Dis. 11, 1738-1741 (2005).

14. Bell, D. M. Public health interventions and SARS spread, 2003. Emerg. Infect. Dis. 10, 1900-1906 (2004).

15. Hufnagel, L., Brockmann, D. \& Geisel, T. Forecast and control of epidemics in a globalized world. Proc. Natl Acad. Sci. USA 101, 15124-15129 (2004).

16. World Health Organisation Writing Group, Non-pharmaceutical interventions for pandemic influenza, international measures. Emerg. Infect. Dis. 12, 81-87 (2006).

17. Hong Kong International Airport. 〈http://www.hongkongairport.com/eng/ aboutus/statistics.html> (2003).

18. Gani, R. et al. Potential impact of antiviral drug use during influenza pandemic Emerg. Infect. Dis. 11, 1355-1362 (2005).

19. Coombes, R. UK stocks up on antiviral drug to tackle flu outbreak. Br. Med. J. 330, 495 (2005)

20. Ferguson, N. M., Mallett, S., Jackson, H., Roberts, N. \& Ward, P. A populationdynamic model for evaluating the potential spread of drug-resistant influenza virus infections during community-based use of antivirals. J. Antimicrob. Chemother. 51, 977-990 (2003).

21. Longini, I. M. J., Koopman, J. S., Monto, A. S. \& Fox, J. P. Estimating household and community transmission parameters for influenza. Am. J. Epidemiol. 115, 736-751 (1982).

22. Cauchemez, S., Carrat, F., Viboud, C., Valleron, A. J. \& Boelle, P. Y. A. Bayesian MCMC approach to study transmission of influenza: application to household longitudinal data. Stat. Med. 23, 3469-3487 (2004).

23. Ferguson, N. M. et al. Planning for smallpox outbreaks. Nature $425,681-685$ (2003).

24. Ferguson, N. M., Fraser, C., Donnelly, C. A., Ghani, A. C. \& Anderson, R. M.
Public health risk from the avian H5N1 influenza epidemic. Science 304 , 968-969 (2004)

Supplementary Information is linked to the online version of the paper at www.nature.com/nature.

Acknowledgements We thank the National Institute of General Medical Sciences MIDAS Program (N.M.F., D.A.T.C. and D.S.B.), the Medical Research Council (N.M.F.), the Royal Society (N.M.F. and C.F.) and the Howard Hughes Medical Institute (N.M.F.) for research funding. We thank B. Schwartz, R.

Robinson, B. Gellin, D. Harper, J. Edmunds, P. Grove, R. Hatchett and members of the MIDAS consortium for useful discussions. We also thank the National Center for Supercomputing Applications (NCSA) and the MIDAS informatics group for computational resources and technical advice.

Author Contributions N.M.F. designed, implemented and ran the model integrated the demographic and disease data sets used, and drafted and revised the text. All other authors edited or commented on the text. D.C. identified collated and processed some demographic and travel data sets used and provided input on model assumptions. C.F. performed analytical modelling that aided the verification of the simulation and gave suggestions on model parameterization. J.C.C. assisted with the collation of demographic and travel data sets. P.C.C. assisted with provision of high performance computing facilities and technical advice. D.S.B. provided input into model design and assumptions, advised on the presentation of results and assisted with data collection.

Author Information Reprints and permissions information is available at npg.nature.com/reprintsandpermissions. The authors declare no competing financial interests. Correspondence and requests for materials should be addressed to N.M.F. (neil.ferguson@imperial.ac.uk). 